\title{
Urgences
}

\section{De la mimonote à la pseudonote (sur la note de pastiche)}

\section{Daniel Bilous}

Numéro 31, mars 1991

Poétique de la note

URI : https://id.erudit.org/iderudit/025634ar

DOI : https://doi.org/10.7202/025634ar

Aller au sommaire du numéro

Éditeur(s)

Urgences

ISSN

0226-9554 (imprimé)

1927-3924 (numérique)

Découvrir la revue

Citer cet article

Bilous, D. (1991). De la mimonote à la pseudonote (sur la note de pastiche).

Urgences, (31), 32-43. https://doi.org/10.7202/025634ar d'utilisation que vous pouvez consulter en ligne.

https://apropos.erudit.org/fr/usagers/politique-dutilisation/ 


\section{De la mimonote à la pseudonote (sur la note de pastiche)}

\section{par Daniel Bilous}

\section{L'imitation, un mimorécit}

À l'instar de la mimésis classique, qui est imitation de la nature, l'imitation d'un écrit relève - au départ du moins d'un projet représentatif. Dans la pureté du geste mimétique, il s'agit ici et là d'offrir à la lecture un substitut si possible parfait de l'objet imité, que celui-ci soit non verbal: physionomie, paysage, voire casquette ( $c f$. Flaubert), ou qu'il soit entièrement fait de mots (les Mémoires de Saint-Simon, par exemple, chez Proust ${ }^{1}$ ). Si l'on peut parler de l'effet de réel d'une description, l'on doit aussi pouvoir parler de l'effet de réel d'une mimésis verbale, actif notamment dans le moindre apocryphe réussi. La perfection implique une totale interchangeabilité entre les termes de la relation mimoscripturale: l'écrit imitatif - nous dirons ici mimécrit - vise une transparence optimale, au prix de laquelle il s'offre comme une doublure de sa cible 2 .

Poussant jusqu'à la théorie le parallèle entre le récit et l'imitation, il devient loisible de présenter cette dernière

1 Marcel Proust, L'affaire Lemoine dans les Mémoires de Saint-Simon, in Pastiches et mélanges.

2 En quoi l'imitation se rapprocherait de la traduction interlinguistique, comme l'avance pertinemment Annie Brisset: « Abstraction faite du changement de code, la traduction est dans son principe une operation absolument mimétique puisqu'elle tend à préserver la spécificité à la fois sémantique et expressive de l'original. Elle vise donc à produire un double du texte de départ ou plus exactement une doublure: le texte traduit est idéalement pris pour l'original et, à défaut, il est lu comme un original [...] La traduction est donc le plus souvent pratiquée comme une opération de la transparence * (Annie Brisset, « La traduction comme transformation para-doxale ", Texte, Toronto, $n^{\circ}$ 4,1985, p. 192. Tant pour la traduction que pour l'imitation, il faut relativiser un tel propos: cette transparence du geste de reprise, qui le rend indecelable à la lecture, n'est que l'une des postulations formelles, l'autre étant la tendance contraire a indexer la pratique. Dans son article "A l'ombre du pastiche", Texte, $n^{\circ} 2$, 1983, Pierre Laurette insiste sur la " spécularité bizarre " du pastiche, et dans le même sens, nous-même avons montré naguère, dans le pastiche, le permanent conflit entre un effet de duplication et un effet de distinction textuelle (Daniel Bilous, "L'antitexte mimétique", Texte \& Antitexte, Actes du ler Colloque International du Centre de Narratologie Appliquee (Cahiers de narratologie, Nice, $\left.n^{\circ} 1,1987\right)$. 
comme une sorte de mimorécit. Par ce terme, nous entendons ce récit spécial dont les «personnages " sont les écrivains imités, engagés dans des "actions" particulières: leurs œuvres, présentées en général sous forme d'extraits où se reconnaît leur style. Qui dit mimorécit dit aussi mimonarrateur, instance dont relève l'énonciation d'un tel récit. Le mimonarrateur désigne l'imitateur en tant qu'il accrédite le rapport entre performance mimétique et auteur imité.

Par ailleurs, la théorie du texte nous le rappelle: il ne faut pas confondre récit et écriture. En deçà du narrateur présent ou implicite se tient une instance dont, en dernier ressort, tout relève: le scripteur, responsable de l'élaboration et de l'agencement des signes, c'est-à-dire du texte. Dans le champ mimétique, ne pas confondre mimorécit et mimécriture conduit à faire une place au mimoscripteur, comme une instance dont relève l'élaboration, l'agencement des signes imitatifs ${ }^{3}$ et surtout la stratégie et le régime d'imitation. Le mimoscripteur désignera donc ici l'imitateur en tant qu'agent réel de la mimécriture.

Mimonarrateur et mimoscripteur se comportent à l'égard du mimorécit comme le narrateur et le scripteur à l'égard du récit. Le dévoilement de l'instance narratrice, s'il révèle le caractère fictionnel du récit, n'a point pour automatique effet de "déréaliser " entièrement la fiction. Certain réalisme, celui par exemple d'un Balzac ou d'un Stendhal, s'accommode parfaitement de ces "intrusions d'auteur" qui sont en fait autant d'intrusions «de narrateur». Il n'en va pas de même pour l'instance d'écriture, dont le surgissement provoque immanquablement la rupture de l'illusion représentative et, simultanée, l'émergence de tel ou tel des aspects "que la représentation subordonne, naturalise, voire oblitère " 4 . Cet effet, pour lequel Jean Ricardou réserve désormais le concept de «métareprésentation ", serait dans le domaine qui nous occupe une méta-mimo-représentation.

Ce bref aperçu théorique permet de situer la pratique imitative au carrefour de deux stratégies. De l'une, mimore-

3 Gérard Genette (Palimpsestes, coll. «Poétique \#, Seuil, Paris, 1982, p. 88) appelle mimétisme a tout trait ponctuel d'imitation ", et mimotexte tout texte imitatif, en prenant le mot texte dans le sens trivial d'écrit, et sans faire la distinction pragmatique que nous operons entre le narrateur et le scripteur de l'imitation. 4 Jean Ricardou, « Elérnents de Textique (1) ", Conséquences, Paris, $n^{\circ} 10$, p. 5. 
34

présentative, l'exemple canonique est le faux ${ }^{5}$, où mimonarrateur et a fortiori mimoscripteur s'effacent. De l'autre, métamimoreprésentative donc, le pastiche classique donne une idée, puisque s'y affichent en pleine lumière et le mimonarrateur et le mimoscripteur. C'est qu'en ce champ particulier de la pratique artistique, à mystifier l'autre, on risque toujours un peu de s'abuser soi-même; l'on croit s'être rendu maître de l'écriture d'autrui au point de tromper son public, et c'est la fascination du modèle qu'à son insu l'on accepte. Il est tentant pour un imitateur de signifier à son lecteur, à tout le moins, l'inauthenticité de sa performance, au plus, comment il a écrit.

Les manières d'y parvenir sont variées mais le mimoscripteur n'a en tout que deux zones pour délivrer ce message critique: le "paratexte" 6 (dont fait partie la note), et l'écrit même.

Ainsi, l'on peut concevoir une imitation aussi transparente que possible, mais avouée comme telle. La déclaration peut être externe à la performance, avec les titres: « à la manière de...", "comme dirait...", "copie conforme" (ou tout autre intitulé équivalent au niveau du contrat de lecture), et à travers l'apposition d'une double signature. Oxymore de la poétique, le résultat, l'apocryphe déclaré est alors, en quelque sorte, un " exercice de style", que seule la nature des cibles (styles d'écrivains) distingue de la forme inventée par Queneau (styles de discours). L'on peut aussi imaginer une déclaration du caractère mimétique tout interne au mimécrit: ce sera la fameuse saturation mimostylistique, qui définit traditionnellement le pastiche.

Ces deux tactiques relevant de la même stratégie, elles ont une particularité en commun: chacune, selon un principe d'économie sémiotique, dispense, en droit, de mettre en place

5 Le domaine mimétique ne saurait se réduire au secteur des apocryphes. Pour une typologie détaillée des mimécrits, voir Daniel et Nicole Bilous, * La manière deux - (pour une didactique du pastiche) $"$, in Claudette Oriol-Boyer et alii, La réécriture, Grenoble, Ceditel, 1990, p. 125-140; et notre these: Mallarméides - Les réécritures de l'œuvre de Mallarmé - Poétique et critique (à paraître).

6 Gérard Genette, Seuils, coll. « Poétique ", Paris, Seuil, 1987, p. 7. II nous faut d'emblée renvoyer à l'utile précision théorique apportée naguère par Jan Baetens, dans son article * Paratexte $=$ péritexte + épitexte " (Conséquences, $n^{\circ}$ 11, p. 94-96). Et préciser que notre propos concerne exclusivement ici, dironsnous, le périmimotexte, ou paratexte annexé à la performance mimétique, "qui escorte le texte au sein du volume publié " (ibid., p. 94). 
l'autre. Il suffit en effet de mentionner dans la périphérie du mimécrit son caractère second pour faire instantanément lire tous les signes comme autant de signifiants mimétiques. Inversement, nul besoin de faire une telle mention latérale si le pastiche dispose, avec la caricature ${ }^{7}$, de suffisants décalages. Toutefois, la littérature mimétique en témoigne massivement, l'économie n'est pas le souci principal du pasticheur moyen. Tout se passe comme si l'imitateur n'avait de cesse de dévoiler son geste de toutes les façons possibles.

Sans doute faut-il faire une différence entre les deux tactiques évoquées. La charge pastichielle porte en soi des vertus satiriques dont reste en principe exempte la mention périphérique. Mais procéder à une déclaration à la fois externe et interne, c'est lever toute ambiguïté. S'il comptait uniquement sur la saturation des stylèmes qu'il renferme pour se déclarer comme inauthentique, le pastiche courrait certainement un risque au niveau de sa réception. En effet, le nom de l'auteur-cible, qui figure invariablement dans le titre d'un pastiche, crée un horizon d'attente stylistique que le mimécrit va satisfaire ou décevoir. Dès lors, quand la caricature cumule la double fonction et de déclarer l'imitation et de proposer une lecture perverse de la cible, le lecteur pourrait bien n'en rester qu'à la désillusion première. En revanche si, au prix d'une certaine redondance de marques, le lecteur est averti d'emblée (grâce à l'apparat périphérique) du caractère mimoscriptural de la performance, il peut à loisir, dans le mimécrit, savourer des calculs pastichiels souvent fort subtils.

\section{La note de pastiche: un lieu stratégique}

Cette situation optimale de partage des rôles ne se réalise cependant que dans le cadre d'un rapport entre signatures et performance, ou entre intitulé et performance, c'est-à-dire avec le paratexte inaugural, celui qui ouvre la lecture. La note, ordinairement infrapaginale, fait partie du paratexte d'escorte, voire terminal.

Par ailleurs, s'agissant d'un objet si ouvertement duplice, il est clair que la note de pastiche va devoir, en quelque sorte, choisir son camp.

7 Gérard Genette donne ainsi un exemple de «pastiche anonyme ", avec le fragment du roman L'écumoire, de Crébillon, où la taupe Moustache pastiche le style de Marivaux (Palimpsestes, p. 98-99). 
Soit elle vise, de quelque façon que ce soit, l'objet simulé, c'est-à-dire en dernière analyse la cible donnée à reconnaître sous le mimécrit. Soit elle pointe, d'une manière plus ou moins adroite, la simulation de l'objet, c'est-à-dire le mimécrit en tant que tel.

Dans le premier cas, au plan énonciatif, la note émane de l'instance mimonarratrice. Elle intègre une stratégie, disons, de mimovraisemblance ${ }^{8}$ : que le mimécrit soit fidèle ou non, si l'apparat critique qui l'accompagne concerne son inévitable part de fidélité, l'illusion d'authenticité en reçoit un appoint non négligeable, car l'ensemble - performance et notes s'offre dès lors comme la classique édition commentée. Appelons ce premier type la mimonote, puisque sa présence signale simplement que l'imitation, en simulant des notes, a étendu son empire aux marges naturelles de l'écrit.

Avec le second cas, la note relève non moins évidemment du mimoscripteur, selon une stratégie de dévoilement dont l'exercice contrecarre la mimovraisemblance, puisque par elle se dissout l'illusion d'authenticité. Sauf platitude insigne et dont nous n'avons pas d'exemple, le dévoilement apporté par une note de pastiche ne saurait être aussi explicite qu'il l'est avec la première de couverture d'un recueil ou les titres courants. L'on pourrait nommer pseudonote ce second type, eu égard à sa nature un rien perverse: elle semble gloser l'écrit représenté, et c'est le texte réel - le pastiche - qu'elle commente, et signale.

L'analyse qui suit voudrait, sur trois échantillons, montrer comment dans le pastiche l'on glisse de la mimonote, qui est un pastiche de note, à la pseudonote, qui est une note de pastiche. Nos exemples sont tous trois tirés de la série, aussi illustre que méconnue, des À la manière de..., par Paul Reboux et Charles Müller.

\section{Exemple 1: le dévoilement négatif}

Dans le Marcel Proust: un mot à la hâte..., le mimonarrateur décrit allusivement la genèse de l'écriture proustienne, au travers d'un personnage, Swan, que son exquise politesse va rendre intarissable. 
Alors, Swan décida d'écrire un très court billet d'excuse, pour Mme Verdurin, afin de se faire absoudre plus sûrement d'une défection qu'il se reprochait à lui-même; il demanda qu'on lui donnât une plume, de l'encre, du papier.

[...] Comme un caoutchouc tendu qu'on lâche, ou comme l'air entre dans une machine pneumatique entr'ouverte, l'inspiration le cinglait, pénétrait en lui, galopait dans le champ du présent et l'enrichissait de possibilités immédiates au point que, par le chimisme même de sa substance cérébrale, il fixait les reflets des moindres bigarrures d'idées qui jouaient dans ses circonvolutions cervicales, car sa pensée était comme amorcée par un siphon, et l'on pouvait se demander quelle intervention en tarirait le flux; chaque mot éveillait en lui des ombres et des lumières, des nuages fugaces, des silhouettes de personnages un jour entrevus, tout un passé qui, perdu pour d'autres, montait des profondeurs de sa mémoire, lentement pénétré de grâce vivante; comme ces joujoux qui, présentés sous forme de fragments menus et secs, et qui, dès qu'ils sont posés sur la surface de l'eau, se colorent et s'épanouissent, ainsi les feuilles déjà nombreuses qu'il avait couvertes de son écriture présentaient nombre de ces addenda que les écrivains, dans leur parler professionnel, nomment des "ballons", sortes de paragraphes enclos d'un paraphe marginal accroché au point du texte que cette addition doit enrichir. Vers neuf heures du soir, Mme Verdurin s'enquit de ce que faisait Swan. Comme le domestique avait répondu: *Ce monsieur écrit toujours ", elle avait ordonné qu'on lui portât discrètement une nouvelle provision de papier et un plateau chargé d'un repas froid. Le lendemain, à midi, Swan n'avait pas encore achevé sa lettre [...] Désormais, cette sollicitude, de même que celle qui s'étendait à la fourniture du papier, permirent à l'écrivain de ne plus être contraint par des contingences misérables à juguler son inspiration. Peu à peu, les hôtes du salon Verdurin s'accoutumèrent à cette présence durable et constante. "C'est ce que j'ai vu de plus fort depuis les tables tournantes! " avait affirmé $M^{\text {me }}$ de Pataty, au début. Maintenant, l'habitude s'était mise en pantoufles dans les âmes rassises, tandis que Swan écrivait toujours, car la moindre variation atmosphérique suffisait à provoquer un changement de ton dans sa sensibilité, à en modifier lalternance; souvent un trait égaré des éléments dissociés interrompait le rêve qu'il aurait pu taire en plaçant, plus tôt ou plus tard qu'à son tour, tel feuillet détaché et interpolé de la correspondance amicale; mais de 
ces phénomènes naturels, son confort et sa santé ne pouvaient tirer qu'un trouble accidentel assez mince, jusqu'au jour où l'exercice s'emparât d'eux et, permettant leur réalisation plus fréquente et mieux rythmée, remît dans les mains de Swan la possibilité de leur apparition soustraite à la tutelle et dispensée de l'agrément du hasard.

P. S. - Pour assister au moment où Swan achève sa lettre, lire le roman suivant: À L'OMBRE FRUIT DES JEUNES GENS, chapitre: DOUZE ANS APRÈS. ${ }^{9}$

Qu'on s'en avise, pourtant: rien dans le récit ne permet cette interprétation qui assimile un personnage, substitut de Swann (à une lettre près), et l'écrivain-cible. Dans le romancible, en dépit de la communauté des prénoms, il ne viendrait (ou ne devrait venir) à l'idée de personne d'assimiler à l'étourdie le narrateur Marcel et l'écrivain Marcel Proust. Seule la connaissance critique que nous pouvons avoir de la façon dont ce dernier écrivait ses romans induit à pareil saut, au prix d'un parallèle qui risquerait fort de ne révéler qu'une simple allusion, si évidente parût-elle au proustologue.

N'était cette mimonote étrange, "pseudo-éditoriale», pour le dire selon Genette ${ }^{10}$. Note d'éditeur au prime abord, elle se révèle, au second coup d'œil, un très efficace opérateur de transmutation du texte. Certes, le contenu ouvertement satirique, aggravant la caricature, dissipe toute méprise. Mais plus encore il faut prendre garde à la forme de l'ajout. Par ce "P. S.» si anodin, Reboux change rétrospectivement d'une façon radicale toute notre lecture de l'extrait romanesque. En celui-ci et jusque là, Swan était le personnage dont un narrateur (probablement le Marcel de La recherche) relatait les embarras épistolaires. Le Post-Scriptum fait du récit qui précède la... lettre même que ce personnage écrivait, et qu'évoquait le récit! Certes, par là, l'imitateur ne favorise pas peu l'assimilation du narrateur et de l'auteur-cible. Mais cette espèce de synecdoque impossible (qu'un seul et même segment narratif soit à la fois le tout et sa partie), dont la note est le site et qui préfigure de loin certaines manœuvres néoromanesques, joue ici, pour une part, comme un signal d'inauthenticité de l'écrit, conférant un appoint structural, aussi puissant qu'il est discret, aux plus voyantes saturations.

9 Paul Reboux, À la manière de..., quatrième série, Paris, Grasset, 1925, p. 85-88.

10 Gérard Genette, Seuils, p. 296. 


\section{Exemple 2: le dévoilement positif}

La pseudonote peut ainsi indiquer ce que le pastiche n'est pas.

Elle peut aussi révéler ce que, spécifiquement, il est. Soit, par les mêmes, le Cleopastre de Jean Racine. Gérard Genette le décrit à bon droit comme un "texte apocryphe accompagné de notes allographes fictives dues à la plume de "M. Libellule, professeur de troisième classe au lycée de Romorantin " ${ }^{11}$, improbable instance pseudo-éditoriale derrière laquelle se dissimulent nos mimonarrateurs. Or, comme tel, le pastiche assume l'apparence d'une classique édition à l'usage des classes. "C'est une savoureuse charge de l'annotation scolaire telle qu'on la pratiquait encore, ou déjà, dans cette fín de siècle", ajoute l'auteur de Seuils, qui voit dans cette "simulation ouvertement satirique", "en un sens, un investissement [fonctionnel] plus fort" de la note par le «discours auctorial »: ici, celui des satiristes.

C'est peut-être faire la part trop belle, précisément, à la caricature, sans s'aviser du problème de concurrence qu'un tel double ciblage ne manque pas de poser. Il est sensible dès les premières pages ${ }^{12}$ :

\section{ACTE I}

La scène est à Alexandrie, dans les cabinets qui sont entre les appartemens d'Antoine et ceus de Cléopastre.

SCÈNE I

CLEOPASTRE, ZOE

ZOÉ

Ouy, Madame, espérez un destin plus paisible.

Auguste à vos attraits ne doibt estre insensible.

\section{CLÉOPASTRE}

II se peut, et mon cœur, de sa flame occupé,

Contre toute rayson voudroit croire à Zoé 1 .

1. Zoé. - Le dictionnaire de Littré (T. IV, p. 2554) donne de ce mot la définition suivante: terme de zoologie; sorte de crustacé presque microscopique.

11 On lit, en haut de la page des dramatis personæ, “ Cléopastre / Premier acte inédit d'une pièce inconnue jusqu'à ce jour/colligé, annoté et interpolé/Par M. LIBELLULE [insigne des palmes académiques]/Professeur de troisième classe au Lycee de Romorantin "..

12 Paul Reboux et Charles Müller, À la manière de..., troisième série, Grasset, Paris, 1913, p.4-5. 
Mais l'ingrat depuys hier ${ }^{1} \mathrm{de}$ ce cabinet l'hoste Se dérobe au destin qui nous mit côte-à-coste ${ }^{2}$, Et, détournant les yeus d'un object trop épris, Du plus dous des thrésors veult ignorer le prix. En vain, pour engager ${ }^{3}$ un amant si rebèle, Aux suppliques d'Antoine ay-je resté cruèle. Auguste me dédaigne et, de gloire embrasé, Ne répond à mon feu que par un feu glacé 4 .

1. Hier. - Il est a remarquer que ce vocable peut avoir deux pieds ou un seul, comme c'est ici le cas.

2. Cóte-â-coste. - Au dix-septième siècle on avait coutume, dans les mots tels que: cóte, faute, aéronaute, de remplacer l'accent circonflexe par un $\mathrm{s}$.

3. Engager. - L'infinitif est ki employé absolument. Aujourd'hui ce terme, non suivi de complément indirect, ne s'usite plus guère que dans la locution: j'ai engagé une cuisinière. Encore faut-il distinguer s'il s'agit d'une domestique que l'on prend en service, ou d'un posle culinaire que l'on porte au Mont-de-Piété.

4. Glacé. - Cf.: Phàre $(1,3)$.

El dérober au jour une flamme si noire.

La cible principale du pastiche n'est-elle pas, plus que Racine même, la cuistrerie philologico-culturelle des magisters qui "l'expliquent", l'alourdissent et en dégoûtent le jeune public? Le dispositif de la note infrapaginale a pour effet de déplacer une bonne part de l'impact satirique vers son auteur supposé. D'où l'urgence d'un recentrage du tir, auquel pourvoit l'ultime note:

[...] C'est la Reyne, elle approche et sous quelque moment...

Suys-moy, cher Exutoire, en mon app... ${ }^{1}$

RACINE

1. App... Ici s'arrête le manuscrit. Comment Racine aurait-il achevé le vers, les érudits en discuteront toujours. Adhuc sub judice lis est. Ce que nous pouvons affirmer, d'après ce que nous connaissons de la pièce, c'est qu'elle se serait de tous points conformée aux règles aristotéliciennes. Les chefs-d'œuvre du theâtre classique sont tous fondus dans le même moule. Au theatre, le public applaudit toujours les moules. Les spectacles contemporains en fournissent maints exemples. 13

Salutaire mise au point. Sous le discours obvie de la glose: un commentaire sur l'universalité des règles de la dramaturgie classique, vient pointer, par syllepse, le second référent du motif: ce "moule» dans lequel les pasticheurs, aussi bien, fondent la performance ${ }^{14}$ qu'on vient de lire. Le

13 Id., p. 16.

14 Même si surgit, par calembour, un nouveau sens de " moule " : niaiserie. 
discours des mimoscripteurs suggère que nous n'avons point affaire à deux sens différents du même mot, mais à deux référents distincts pour un seul concept. "Originaux" et "apocryphe" sont par là renvoyés aux mêmes secrets de fabrication. À une rhétorique.

\section{Exemple 3 : le dévoilement descriptif}

Le dernier échantillon nous fait encore monter d'un degré dans l'autodésignation du procès pastichiel dans et par la pseudonote. Il ne s'agit plus seulement d'indiquer sur le mode négatif ou même positif, la nature allographe ou mimétique de l'œuvre soumise à lecture. Il s'agit en sus de permettre au lecteur l'accès aux mécanismes mêmes par lesquels s'élabora la performance, dans un élan de généreuse révélation.

Un dispositif un peu plus complexe est nécessaire, dont les Sonnets de Mallarmé - alias Reboux et Müller offriront la mesure ${ }^{15}$. On le sait, pour traiter ce poète, les pasticheurs imaginent un micro-recueil, avec deux pièces assorties d'une bien curieuse «Glose»:

SONNETS

Quand le vaticinant erratique, au larynx Dédaléen, divague en sa tant dédiée Et de l'Absent manie avant tout radiée Pour de l'insaisissable animer la syrinx,

Ô n'être qu'aboli le mystère du sphinx Par qui du clair-obscur l'âme est congédiée!

Ô chevaucher, vers la victoire irradiée, Aveugle, et de ses yeux exorbité, le lynx!

Hypogéenne telle énigme la Pythie, Ambage non pas un d'où l'inconnu dévie, J'ai de l'impénétrable approfondi l'azur,

Et, ténébral sitôt hiéroglyphique cygne, Qu'obstructif en son vide ombre un déléatur, J'offusque, triomphal, le néant qui m'assigne.

15 Paul Reboux et Charles Müller, À la manière de..., troisième série, p. 141149. Nous n'en citons qu'un extrait. 
42

II

Orgueil du grand sitôt en extase cabré,

$[\cdots]$

\section{GLOSE}

Un groupe d'érudits prépare une traduction française des œuvres de Stéphane Mallarmé. Cette entreprise, en raison des recherches qu'elle nécessite, n'aboutira pas, sans doute, avant de longues années. Nous ne pouvons aujourd'hui donner au lecteur que la traduction du premier de ces deux sonnets:

Quand le vaticinant erratique, au larynx dédaléen, divague, en sa manie tant dédiée et avant tout radiée de l'absent pour animer

le syrinx de l'insaisissable
Quand le poète prophète qui ne sait où il va et dont la parole égare divague, en sa folie si coutumière et qui, avant d'exister, se retranche même de ce qui n'existe pas, lorsqu'il va souffler dans une flûte sans son [...]

Les cryptographes ne se sont pas jusqu'ici mis d'accord sur le sens du deuxième sonnet. Certains proposent une version, mais nous respectons trop nos lecteurs pour la leur mettre sous les yeux. "

La Glose se donne d'abord pour une mimonote où, sur le modèle déjà breveté du Racine, les satiristes s'en prennent autant, sinon plus qu'au poète lui-même, aux exégètes besogneux du verbe mallarméen. Un peu d'attention suffit pour y voir une parfaite machine à lire non le sonnet mallarméen mais bien le pastiche qui en donne ici l'image (passablement chargée, on en conviendra). Il n'est que de comparer soigneusement les versions du sonnet, et d'emblée s'aviser qu'il y en a non pas deux, mais bien trois. À la version canonique succèdent en effet, mises en miroir par la disposition juxtalinéaire, deux types de reformulations.

Colonne de droite, bien sûr, c'est la «traduction française", révélatrice de tant d'obtuseté calculée. Dans la paraphrase assassine, la prose ravale toute l'expression, toute image 
s'aplatit, toute étymologie s'évente. Le seul vocable à demeurer inchangé est: "divague", sans doute pour prendre le poète à un piège qu'il avait d'ailleurs construit (et, partant, dominé), en intitulant l'un de ses livres: Divagations. Et «le syrinx de l'insaisissable" devenant "une flûte sans son", montre assez qu'il n'est pas question de communier jamais avec l'univers mental de ce "vaticinant». On le regarde, inexorablement, de l'extérieur, avec les yeux de «la tribu».

Mais colonne de gauche, non moins, se déploie une réécriture syntaxique du sonnet. Le poème est mis à plat, les syntagmes utiles reformés, et rétabli, «l'ordre-normal-desmots-dans-la-phrase». Ainsi, dans le premier quatrain, le lecteur s'avise qu'il doit remonter «manie" au second vers s'il veut construire la phrase. (Le malheur est que cette mise à plat met aussi le poème... en pièces; satire oblige).

Donc si, à droite, on a bien une paraphrase sémantique, c'est la traduction d'un recodage, qui vise non le poème, mais sa mise à plat, sa forme déjà médiatisée.

C'est dire que la pseudonote ne possède pas seulement une vertu critique vis-à-vis de la cible, sur un mode allométatextuel, qui doublerait les effets de loupe de la caricature. Elle déploie aussi une puissante aptitude à pointer les propres mécanismes du pastiche, sur le mode autométatextuel, ou plutôt, s'agissant d'un mimécrit: métamimotextuel. La lire, c'est apprendre indirectement comment est fait le pastiche, puisque aidé du dispositif triple (poème, segmentation, paraphrase), l'on peut sans trop d'efforts recenser les mallarméismes sélectionnés par les mimoscripteurs ${ }^{16}$.

Sans cacher ses préférences pour les calculs plus économes, Genette avance qu'au-delà des grossissements propres au pastiche satirique, "la pratique caricaturale s'accompagne presque constamment, par voie paratextuelle (préfaces, notes, interviews, etc.), d'un commentaire chargé de mettre les points sur les $i{ }^{17}$. Nos trois exemples l'attestent: certains pastiches sont un rien plus subtils que ne le laisse entendre un tel propos. Peut-être l'essentiel est-il, pour le mimoscripteur, d'apposer sa griffe quelque part, en façon de signature, au moins autant que de réussir, à coups de griffes, une lourde charge. Cet essentiel-là, parfois, se lit en note. 